\title{
RMNv2: Reduced Mobilenet V2 for CIFAR10
}

\author{
Maneesh Ayi \\ Department of ECE \\ Purdue School of Engineering and Tech. \\ Indianapolis, USA \\ maayi@iu.edu
}

\author{
Mohamed El-Sharkawy \\ Department of ECE \\ Purdue School of Engineering and Tech. \\ Indianapolis, USA \\ melshark@iupui.edu
}

\begin{abstract}
In this paper, we developed a new architecture called Reduced Mobilenet V2 (RMNv2) for CIFAR10 dataset. The baseline architecture of our network is Mobilenet V2. RMNv2 is architecturally modified version of Mobilenet V2. The proposed model has a total number of parameters of $1.06 \mathrm{M}$ which is $52.2 \%$ lesser than the baseline model. The overall accuracy of RMNv2 for CIFAR10 dataset is $92.4 \%$ which is $1.9 \%$ lesser than the baseline model. The architectural modifications involve heterogeneous kernel-based convolutions, mish activation, etc. Also, we include a data augmentationtechnique called AutoAugment that contributes to increasing accuracy of our model. This architectural modification makes the model suitable for resource-constrained devices like embedded devices, mobile devices deployment for real-time applications like autonomous vehicles, object recognition, etc.

Index Terms-MobilenetV2 architecture, Convolution Neural Networks (CNN), Deep Neural Networks (DNN), CIFAR-10, Pytorch.
\end{abstract}

\section{INTRODUCTION}

Convolution neural networks (CNN) come into the picture in the field of Computer Vision with the introduction of AlexNet [6]. It proves to be a trendsetter in Computer vision platform by winning the Imagenet challenge: ILSVRC 2012 [7]. Then over the period of time, there have been many new architectures that are much bigger and complicatedintroduced to achieve higher accuracy. One of that kind is VGG net [11].[15][16] also follow the same trend with increased accuracies. However, implementing these networks in resource-constrained environments like embedded devicesis very difficult. Due to insufficient memory, limited computational capacity real-time implementation of bigger networks is not possible in these devices. So, there is a needto develop smaller networks that require less computation, less memory while maintaining competitive accuracy. This paper aims to introduce a new $\mathrm{CNN}$ architecture that gives out better accuracy with low model size.

Section-2 describes prior works in building small models. Section-3 describes the developed network RMNv2 architecture and Section- 4 talks about the experiments carried out and results of that experiment. Finally, Section- 5 concludes the paper.

\section{PRIOR WORK}

There has been active research going from the past several years in designing small models. This includes either
TABLE I: Mobilenet V2 Baseline

\begin{tabular}{c|c|c|c|c|c} 
Input & operator & t & c & n & s \\
\cline { 2 - 6 } $224^{c} \times 3$ & Conv2D & - & 32 & 1 & 2 \\
$112^{c} \times 32$ & Bottleneck & 1 & 16 & 1 & 1 \\
$112^{c} \times 16$ & Bottleneck & 6 & 24 & 2 & 2 \\
$56^{c} \times 24$ & Bottleneck & 6 & 32 & 3 & 2 \\
$28^{c} \times 3 L$ & Bottleneck & 6 & 64 & 4 & 2 \\
$14^{c} \times 64$ & Bottleneck & 6 & 96 & 3 & 1 \\
$14^{c} \times 96$ & Bottleneck & 6 & 160 & 3 & 2 \\
$7^{<} \times 160$ & Bottleneck & 6 & 320 & 1 & 1 \\
$7^{c} \times 320$ & Conv2D & - & 1280 & 1 & 1 \\
$7^{<} \times 1280$ & AvgPool & - & - & 1 & - \\
$1^{c} \times 1280$ & Conv2D & - & $\mathrm{k}$ & - &
\end{tabular}

compressing a large neural network or designing small networks directly. There are certain techniques to compress a trained network like quantization[8], hashing[9], pruning, vector quantization, and Huffman Encoding[10]. Another method called distillation [12] which uses large network to teach small network. And also there are several techniques [13][14] that are used to compress the pre-trained neural network. Another approach is to design small networks directly for training. SqueezeNet[17] and SqueezeNext[18]are some of the examples of this approach. Most of these small networks mainly focus on small size but they do not focus on speed. Mobilenets[1] are introduced which are designed using depthwise separable convolutions and pointwise convolutions. They focus on both speed and accuracy. Our network is designed from Mobilenet V2[2].These networks try to reduce the number of computations required making it suitable for deploying in mobile and embedded devices.

\section{RMNV2 ARCHITECTURE}

The proposed $\mathrm{RMNv} 2$ architecture is a $\mathrm{CNN}$ architecture inspired by Mobilenet V2, Heterogenous kernel-based convolution and mish activation function. Table-1 shows the baseline Mobilenet V2 architecture. In this section, we describe the detailed architecture of the proposed model.

\section{A. Disabling downsampling layers}

We have disabled some downsampling layers in our architecture. Its because the original Mobilenet V2 architecture is proposed for Imagenet dataset with an input image size of $224 \times 224 \times 3$. In order to make it compatible

This is the author's manuscript of the article published in final edited form as: 


\begin{tabular}{l|c|c|c|c|c} 
TABLE II: Strides changed from 2 to 1 \\
\cline { 2 - 6 } \multicolumn{1}{c|}{ Input } & operator & t & c & n & s \\
\hline $224^{2} \times 3$ & Conv2D & - & 32 & 1 & 1 \\
$112^{c} \times 32$ & Bottleneck & 1 & 16 & 1 & 1 \\
$112^{<} \times 16$ & Bottleneck & 6 & 24 & 2 & 1 \\
$56^{c} \times 24$ & Bottleneck & 6 & 32 & 3 & 1 \\
$28^{c} \times 32$ & Bottleneck & 6 & 64 & 4 & 2 \\
$14^{c} \times 64$ & Bottleneck & 6 & 96 & 3 & 1 \\
$14^{c} \times 96$ & Bottleneck & 6 & 160 & 3 & 2 \\
$7^{c} \times 160$ & Bottleneck & 6 & 320 & 1 & 1 \\
$7^{c} \times 320$ & Conv2D & - & 1280 & 1 & 1 \\
$7^{c} \times 1280$ & AvgPool & - & - & 1 & - \\
$1^{c} \times 1280$ & Conv2D & - & $\mathrm{k}$ & - &
\end{tabular}

to CIFAR10 dataset we have disabled some downsample layers by simply replacing strides 2 with stride 1 . Table- 2 shows us the disabled downsampled Mobilenet V2 architecture. The values highlighted in yellow color are the changes done with respect to base architecture.

\section{B. Replacing Bottlenecks with HetConv Blocks:}

Bottlenecks play a crucial role in Mobilenet V2. It helps in preventing from non-linearity destroying a lot of useful information. We can see the bottlenecks used in Mobilenet

V2 in the following figure, (a) Regular

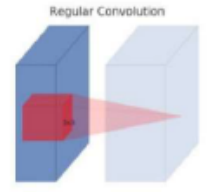

(c) Separable with linear bottleneck

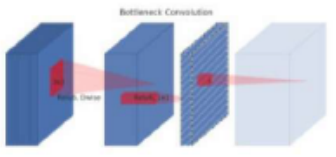

(b) Separable

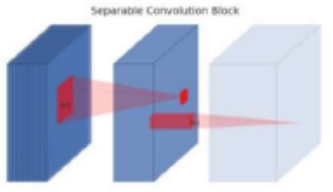

(d) Bottleneck with expansion layer

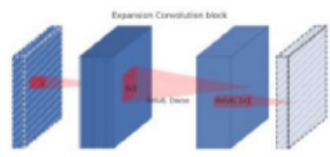

Fig. 1: Bottlenecks used in Mobilenet V2

These bottlenecks shown uses a homogeneous kernel of size 33 . In our experiment we replace this homogeneous kernel with Heterogeneous Kernel[3] of different sizes. The main idea of replacing this bottleneck with hetconv block is explained below,

Let us assume an input feature map of size $A_{i} \quad A_{i \times} C_{i}$ where $A_{i}$ is input feature map spatial height and width. Let $C_{i}$ is the number of input channels. Also consider output feature map of size $A_{0} A_{0} \quad C_{0}$ where $A_{0}$ is output feature map spatial height and width. $C_{o}$ is the number of output channels. An output feature map is obtained by multiplying $C_{o}$ filters with size $k \quad k$ $C_{i}$ where $\mathrm{K}_{\mathbf{C}}$ is $\backslash$ kernel Size. For depthwise and pointwise convolutions applied in Mobilenet V2, the total computational cost will be,

$$
\text { cost }=A_{i} \times A_{i} \times C_{i} \times k \times k+C_{i} \times C_{o} \times A_{o} \times A_{o}(
$$

There is a need to optimise parameter ' $\mathrm{k}$ '. Mobilenet V2 uses homogeneous kernel of size 33 . In heterogeneous kernel, we use variable kernel sizes to reduce the number of parameters without much compromise in accuracy. In

HetConv, we set a variable $P$ which controls the number of different types of kernels. For part $\mathrm{P}$, a fraction of $1 \div p$ willbe of kernel $k \times k$ size and remaining $(1-(1 \div p))$ will be of 1 $\times 1$ kernel size. So, the computational cost for kernel $k \times k$ at Layer $\mathrm{L}$ is,

$$
\operatorname{cost1}=\left(A_{i} \times A_{i} \times C_{i} \times C_{o} \times k \times k\right) \div p
$$

The Computational cost for $1 \times 1$ kernel will be,

$$
\text { cost2 }=\left(A_{o} \times A_{o} \times C_{o}\right) \times\left(C_{i}-\left(C_{i} \div p\right)\right)
$$

Total cost will be,

$$
\cos t=\cos t 1+\cos t 2
$$

The total reduction when compared to standard convolution is,

$$
R_{\text {Hetconv }}=(1 \div p)+\left((1-(1 \div p)) \div k^{2}\right)
$$

In the above equation, if we put $\mathrm{p}=1$ it becomes a

standard convolution filter The architectural representation of our network is shown in figure-2. In the architecture, we can

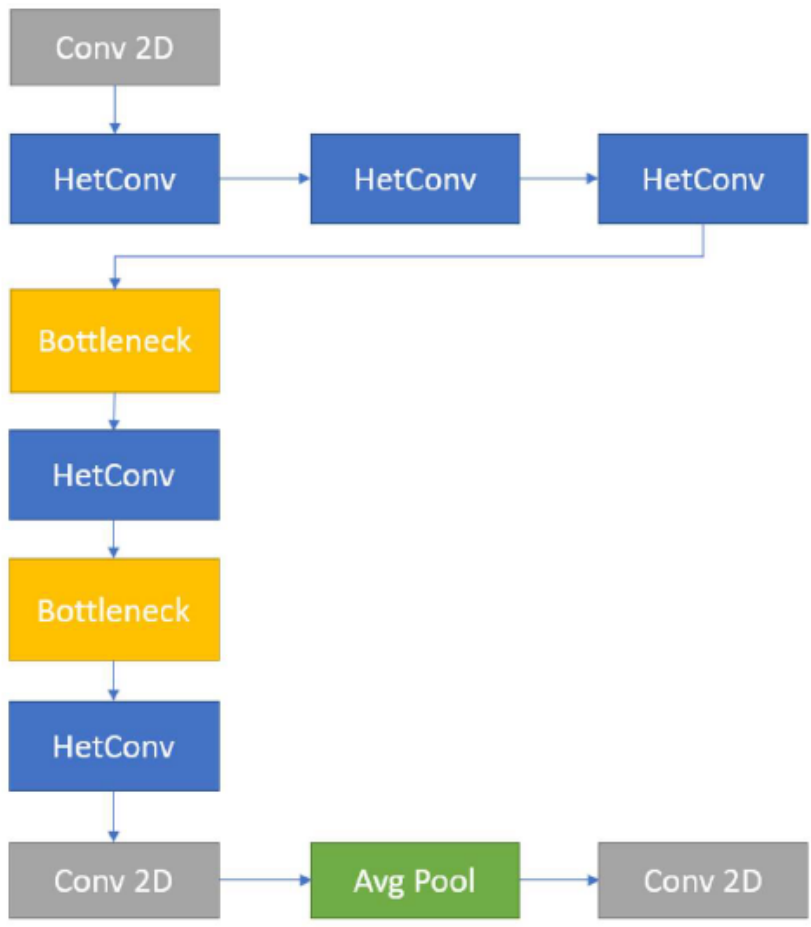

Fig. 2: RMNv2 Architecture

see that we have replaced bottleneck layers with hetconv blocks. The mathematical explanation for this replacement is shown above. We haven't replaced the bottleneck layers where we require downsampling in the network. 


\section{Mish activation function}

Non Linearity is an important concept in a neural network which is introduced through an activation function. Some of the widely used activation functions are ReLU (Rectified Linear Units), TanH (tan Hyperbolic), Swish activationfunction, etc. In our network, we used another activation function called Mish activation function[4]. It is defined as,

$$
f(x)=x \times\left(\tanh \left(1+e^{x}\right)\right)
$$

where $\tanh \left(1+e^{x}\right)$ is a softplus activation. The graph of the mish activation function is shown below,

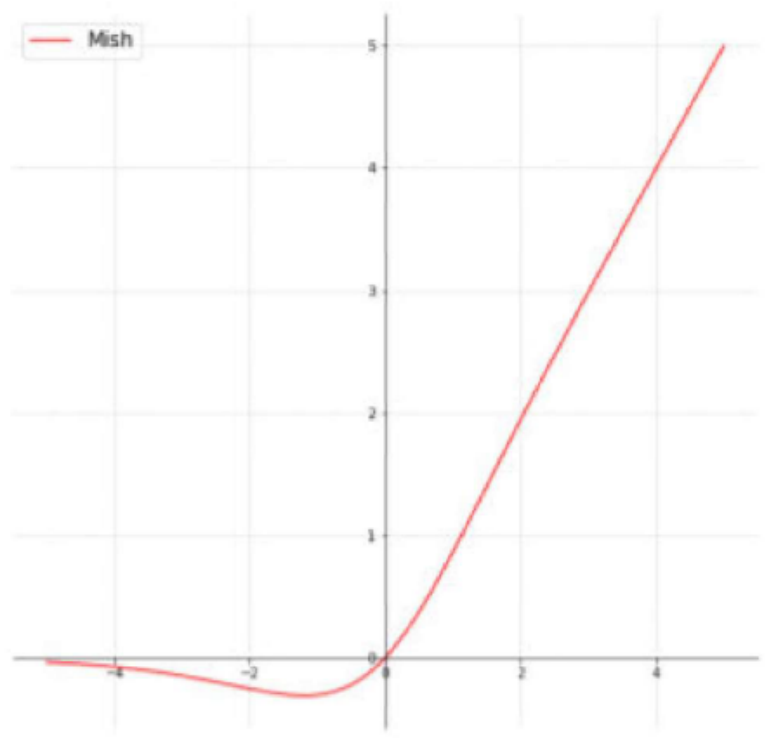

Fig. 3: Mish Activation Function

\section{AutoAugmentation}

For modern image classifiers, data augmentation plays a crucial role in altering the performance of a classifier. So, effective data augmentation techniques are required to boost up the accuracy. AutoAugmentation[5] is one of the implementations which automatically searches for improved data augmentation policies. In a policy, there are many subpolicies designed. Sub policy consists of two functions, one function is an image processing function like rotate, translate, etc. and other is probability or magnitude of that image processing function applied. This advancement in data augmentation bolstered our proposed network by increasing its accuracy.

\section{HARDWARE AND SOFTWARE USED}

- Intel i7-8700 processor with 32 GB RAM.

- Nvidia Geforce GTX 1080Ti GPU.

- Python version 3.6.7.

- Spyder version 3.6.

- Pytorch version 1.0.

- Livelossplot (Loss and accuracy visualization).
TABLE III: Comparison of Various Results between Baseline and Proposed Network

\begin{tabular}{|l|l|l|l|}
\hline \multicolumn{4}{|c|}{ Comparision of Various Results } \\
\hline Model & Model Accuracy & \#parameters & $\begin{array}{l}\text { Model Size(in MB) } \\
\text { Baseline }\end{array} 9.1$ \\
\hline RMNv2 (Ours) & $92.4 \%$ & $22378 \mathrm{M}$ & 9.1 \\
\hline
\end{tabular}

\section{RESULTS}

We have trained our network using PyTorch framework using Nvidia Geforce GTX 1080Ti GPU. The network is trained with Stochastic Gradient descent (SGD) optimizer with a variable learning rate of $0.1,0.01,0.001$ keeping a total number of epochs up to 200. For training the network, we have used a batch size of 128 and for test epoch we useda batch size of 64 . The baseline Mobilenet V2 after disabling the downsampling layers gives us the following results shown in Figure,

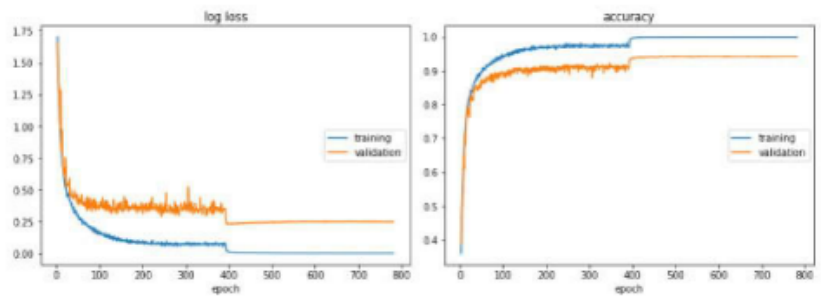

Fig. 4: Baseline Results

we have used the same optimiser, learning rates and batch sizes that are used to train baseline network. The accuracy and loss curves of our network (RMNv2) are shown in Figure-6. For our network we assigned the number of groups, $\mathrm{p}$ value to 4 diagrammatically it is shown as,

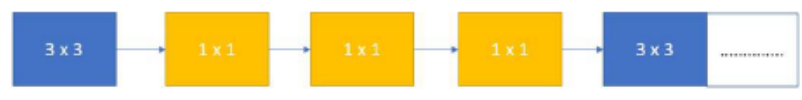

Fig. 5: Heterogeneous Kernel for $\mathrm{P}=4$

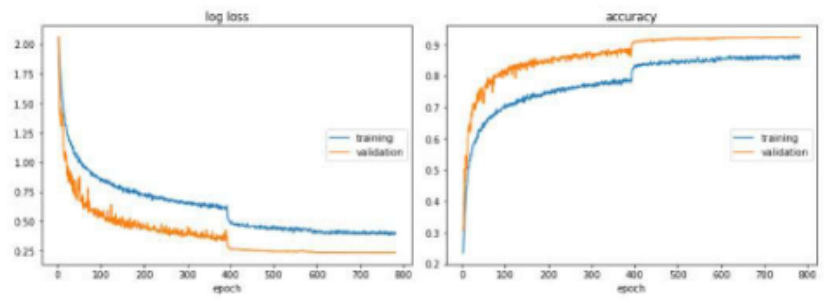

Fig. 6: RMNv2 Results

These curves are plotted using LiveLossPlot Visualization library. Table- 3 compares various results like model accuracy, number of parameters, model size, etc.

Table- 4 shows us the time taken by each model to complete one epoch as well as the whole training for 200 epochs. 
TABLE IV: Comparison of Time between Baseline and Proposed Network

\begin{tabular}{|l|l|l|}
\hline \multicolumn{2}{|c|}{ Comparision of Time } \\
\hline Model & For one epoch(in min) & For Complete training(in hr) \\
\hline Baseline & 1.9097 & 6.7 \\
\hline RMNv2 (Ours) & 0.7621 & 2.7 \\
\hline
\end{tabular}

In the appendix section, we have shown model summary for both baseline and RMNv2. In that table, we can clearly see how many parameters produced by each layer as well as the output shape produced by the input tensor multiplying with different filters.

\section{CONCLUSION}

The results show that our network Reduced Mobilenet V2 (RMNv2) requires fewer computations, lesser time than the original model with not much decrease in accuracy, $1.9 \%$. It is clearly shown that replacing bottlenecks with HetConv layer helped us in decreasing the model size by $52.2 \%$. In order to boost up the accuracy, we replaced ReLU6 activation with Mish activation and we also used effective data augmentation technique like Autoaugmentation whichhelped us in increasing accuracy to $92.4 \%$. Altogether it forms up a new architecture called Reduced Mobilenet V2 (RMNv2). The optimised model size with competing accuracy makes the RMNv2 model suitable for deploying in resource-constrained devices like Embedded devices, Mobile devices, etc. For future work, one can try further model reduction techniques like model pruning, quantization and huffman encoding etc. And also we can try to improve the accuracy by implementing better augmentation techniques, transfer learning and better architectural changes like changing convolutions etc. We can also check the real-time inference of RMNv2 in embedded devices to demonstrate its performance in real-world applications.

\section{REFERENCES}

[1] Howard, Andrew G., et al. "Mobilenets: Efficient convolutional neural networks for mobile vision applications." arXiv preprint arXiv:1704.04861 (2017).

[2] Mark Sandler, Andrew Howard, Menglong Zhu, Andrey Zhmoginov, Liang-Chieh Chen. "MobileNetV2: Inverted Residuals and Linear Bottlenecks." arXiv preprint arXiv:1801.04381v4 (2019)

[3] Pravendra Singh, Vinay Kumar Verma, Piyush Rai, Vinay P. Namboodiri. "HetConv: Heterogeneous Kernel-Based Convolutions for Deep CNNs" arXiv preprint arXiv:1903.04120v2 (2019)

[4] Diganta Misra, "Mish: A Self Regularized Non-Monotonic Neural Activation Function" arXiv preprint arxiv:1908.08681 (2019)

[5] Ekin D. Cubuk, Barret Zoph, Dandelion Mane, Vijay Vasudevan, Quoc V. Le Google Brain, "AutoAugment: Learning Augmentation Strategies from Data" arXiv preprint arXiv:1805.09501v3 (2019)

[6] A. Krizhevsky, I. Sutskever, and G. E. Hinton, "Imagenet classification with deep convolutional neural networks" In Advances in neural information processing systems, pages 10971105, 2012.

[7] O. Russakovsky, J. Deng, H. Su, J. Krause, S. Satheesh, S. Ma, Z. Huang, A. Karpathy, A. Khosla, M. Bernstein, et al. Imagenet large scale visual recognition challenge, International Journal of Computer Vision, 2015

[8] J Wu, C Leng, Y Wang, Q Hu, and J Cheng "Quantized convolutional neural networks for mobile devices". arXiv preprint arXiv:1512.06473, 2015 .
[9] W. Chen, J. T. Wilson, S. Tyree, K. Q. Weinberger, and Y. Chen. "Compressing neural networks with the hashing trick". CoRR, abs/1504.04788, 2015

[10] S. Han, H. Mao, and W. J. Dally.”Deep compression: Compressing deep neural network with pruning, trained quantization and huffman coding". CoRR, abs/1510.00149, 2, 2015.

[11] Karen Simonyan, Andrew Zisserman. "VERY DEEP CONVOLUTIONAL NETWORKS FOR LARGE-SCALE IMAGE RECOGNITION" arXiv preprint arXiv:1409.1556v6 (2015)

[12] G. Hinton, O. Vinyals, and J. Dean. "Distilling the knowledge in a neural network". arXiv preprint arXiv:1503.02531, 2015.

[13] M. Jaderberg, A. Vedaldi, and A. Zisserman. "Speeding up convolutional neural networks with low rank expansions." arXiv preprint arXiv:1405.3866, 2014.

[14] V. Lebedev, Y. Ganin, M. Rakhuba, I. Oseledets, and V. Lempitsky. "Speeding-up convolutional neural networks using fine-tuned cpdecomposition." arXiv preprint arXiv:1412.6553, 2014.

[15] C. Szegedy, V. Vanhoucke, S. Ioffe, J. Shlens, and Z. Wojna. "Rethinking the inception architecture for computer vision." arXiv preprint arXiv:1512.00567, 2015.

[16] C. Szegedy, S. Ioffe, and V. Vanhoucke. Inception-v4, inception-resnet and the impact of residual connections on learning. arXiv preprint arXiv:1602.07261, 2016

[17] F. N. Iandola, M. W. Moskewicz, K. Ashraf, S. Han, W. J. Dally, and K. Keutzer. Squeezenet: Alexnet-level accuracy with 50x fewer parameters and $1 \mathrm{mb}$ model size. arXiv preprint arXiv:1602.07360, 2016.

[18] Amir Gholami, Kiseok Kwon, Bichen Wu, Zizheng Tai, Xiangyu Yue, Peter Jin, Sicheng Zhao, Kurt Keutzer SqueezeNext: Hardware-Aware Neural Network Design arXiv preprint arXiv:1803.10615v2 
APPENDIX A

MODEL SUMMARIES

\begin{tabular}{|l|l|l|}
\hline Layer & output Shape & Param \# \\
\hline Conv2d-1 & {$[-1,32,32,32]$} & 864 \\
BatchNorm2d-2 & {$[-1,32,32,32]$} & 64 \\
Conv2d-3 & {$[-1,32,32,32]$} & 1,024 \\
BatchNorm2d-4 & {$[-1,32,32,32]$} & 64 \\
Conv2d-5 & {$[-1,32,32,32]$} & 288 \\
BatchNorm2d-6 & {$[-1,32,32,32]$} & 64 \\
Conv2d-7 & {$[-1,16,32,32]$} & 512 \\
BatchNorm2d-8 & {$[-1,16,32,32]$} & 32 \\
BaseBlock-9 & {$[-1,16,32,32]$} & 0 \\
Conv2d-10 & {$[-1,96,32,32]$} & 1,536 \\
BatchNorm2d-11 & {$[-1,96,32,32]$} & 192 \\
Conv2d-12 & {$[-1,96,32,32]$} & 864 \\
BatchNorm2d-13 & {$[-1,96,32,32]$} & 192 \\
Conv2d-14 & {$[-1,24,32,32]$} & 2,304 \\
BatchNorm2d-15 & {$[-1,24,32,32]$} & 48 \\
BaseBlock-16 & {$[-1,24,32,32]$} & 0 \\
Conv2d-17 & {$[-1,144,32,32]$} & 3,456 \\
BatchNorm2d-18 & {$[-1,144,32,32]$} & 288 \\
Conv2d-19 & {$[-1,144,32,32]$} & 1,296 \\
BatchNorm2d-20 & {$[-1,144,32,32]$} & 288 \\
Conv2d-21 & {$[-1,24,32,32]$} & 3,456 \\
BatchNorm2d-22 & {$[-1,24,32,32]$} & 48 \\
BaseBlock-23 & {$[-1,24,32,32]$} & 0 \\
Conv2d-24 & {$[-1,144,32,32]$} & 3,456 \\
BatchNorm2d-25 & {$[-1,144,32,32]$} & 288 \\
Conv2d-26 & {$[-1,144,32,32]$} & 1,296 \\
BatchNorm2d-27 & {$[-1,144,32,32]$} & 288 \\
Conv2d-28 & {$[-1,32,32,32]$} & 4,608 \\
BatchNorm2d-29 & {$[-1,32,32,32]$} & 64 \\
BaseBlock-30 & {$[-1,32,32,32]$} & 0 \\
Conv2d-31 & {$[-1,192,32,32]$} & 6,144 \\
BatchNorm2d-32 & {$[-1,192,32,32]$} & 384 \\
Conv2d-33 & {$[-1,192,32,32]$} & 1,728 \\
BatchNorm2d-34 & {$[-1,192,32,32]$} & 384 \\
Conv2d-35 & {$[-1,32,32,32]$} & 6,144 \\
BatchNorm2d-36 & {$[-1,32,32,32]$} & 64 \\
BaseBlock-37 & {$[-1,32,32,32]$} & 0 \\
Conv2d-38 & {$[-1,192,32,32]$} & 6,144 \\
BatchNorm2d-39 & {$[-1,192,32,32]$} & 384 \\
Conv2d-40 & {$[-1,192,32,32]$} & 1,728 \\
BatchNorm2d-41 & {$[-1,192,32,32]$} & 384 \\
Conv2d-42 & {$[-1,32,32,32]$} & 6,144 \\
BatchNorm2d-43 & {$[-1,32,32,32]$} & 64 \\
BaseBlock-44 & {$[-1,32,32,32]$} & 0 \\
Conv2d-45 & {$[-1,192,32,32]$} & 6,144 \\
BatchNorm2d-46 & {$[-1,192,32,32]$} & 384 \\
Conv2d-47 & {$[-1,192,16,16]$} & 1,728 \\
\hline
\end{tabular}

\begin{tabular}{|l|l|l|}
\hline Layer & output shape & Param \# \\
\hline BatchNorm2d-48 & {$[-1,192,16,16]$} & 384 \\
Conv2d-49 & {$[-1,64,16,16]$} & 12,288 \\
BatchNorm2d-50 & {$[-1,64,16,16]$} & 128 \\
BaseBlock-51 & {$[-1,64,16,16]$} & 0 \\
Conv2d-52 & {$[-1,384,16,16]$} & 24,576 \\
BatchNorm2d-53 & {$[-1,384,16,16]$} & 768 \\
Conv2d-54 & {$[-1,384,16,16]$} & 3,456 \\
BatchNorm2d-55 & {$[-1,384,16,16]$} & 768 \\
Conv2d-56 & {$[-1,64,16,16]$} & 24,576 \\
BatchNorm2d-57 & {$[-1,64,16,16]$} & 128 \\
BaseBlock-58 & {$[-1,64,16,16]$} & 0 \\
Conv2d-59 & {$[-1,384,16,16]$} & 24,576 \\
BatchNorm2d-60 & {$[-1,384,16,16]$} & 768 \\
Conv2d-61 & {$[-1,384,16,16]$} & 3,456 \\
BatchNorm2d-62 & {$[-1,384,16,16]$} & 768 \\
Conv2d-63 & {$[-1,64,16,16]$} & 24,576 \\
BatchNorm2d-64 & {$[-1,64,16,16]$} & 128 \\
BaseBlock-65 & {$[-1,64,16,16]$} & 0 \\
Conv2d-66 & {$[-1,384,16,16]$} & 24,576 \\
BatchNorm2d-67 & {$[-1,384,16,16]$} & 768 \\
Conv2d-68 & {$[-1,384,16,16]$} & 3,456 \\
BatchNorm2d-69 & {$[-1,384,16,16]$} & 768 \\
Conv2d-70 & {$[-1,64,16,16]$} & 24,576 \\
BatchNorm2d-71 & {$[-1,64,16,16]$} & 128 \\
BaseBlock-72 & {$[-1,64,16,16]$} & 0 \\
Conv2d-73 & {$[-1,384,16,16]$} & 24,576 \\
BatchNorm2d-74 & {$[-1,384,16,16]$} & 768 \\
Conv2d-75 & {$[-1,384,16,16]$} & 3,456 \\
BatchNorm2d-76 & {$[-1,384,16,16]$} & 768 \\
Conv2d-77 & {$[-1,96,16,16]$} & 36,864 \\
BatchNorm2d-78 & {$[-1,96,16,16]$} & 192 \\
BaseBlock-79 & {$[-1,96,16,16]$} & 0 \\
Conv2d-80 & {$[-1,576,16,16]$} & 55,296 \\
BatchNorm2d-81 & {$[-1,576,16,16]$} & 1,152 \\
Conv2d-82 & {$[-1,576,16,16]$} & 5,184 \\
BatchNorm2d-83 & {$[-1,576,16,16]$} & 1,152 \\
Conv2d-84 & {$[-1,96,16,16]$} & 55,296 \\
BatchNorm2d-85 & {$[-1,96,16,16]$} & 192 \\
BaseBlock-86 & {$[-1,96,16,16]$} & 0 \\
Conv2d-87 & {$[-1,576,16,16]$} & 55,296 \\
BatchNorm2d-88 & {$[-1,576,16,16]$} & 1,152 \\
Conv2d-89 & {$[-1,576,16,16]$} & 5,184 \\
BatchNorm2d-90 & {$[-1,576,16,16]$} & 1,152 \\
Conv2d-91 & {$[-1,96,16,16]$} & 55,296 \\
BatchNorm2d-92 & {$[-1,96,16,16]$} & 192 \\
BaseBlock-93 & {$[-1,96,16,16]$} & 0 \\
Conv2d-94 & {$[-1,576,16,16]$} & 55,296 \\
BatchNorm2d-95 & {$[-1,576,16,16]$} & 1,152 \\
Conv2d-96 & {$[-1,576,8,8]$} & 5,184 \\
BatchNorm2d-97 & {$[-1,576,8,8]$} & 1,152 \\
\hline
\end{tabular}




\begin{tabular}{|l|l|l|}
\hline Layer & output shape & Param \# \\
\hline Conv2d-98 & {$[-1,160,8,8]$} & 92,160 \\
BatchNorm2d-99 & {$[-1,160,8,8]$} & 320 \\
BaseBlock-100 & {$[-1,160,8,8]$} & 0 \\
Conv2d-101 & {$[-1,960,8,8]$} & 153,600 \\
BatchNorm2d-102 & {$[-1,960,8,8]$} & 1,920 \\
Conv2d-103 & {$[-1,960,8,8]$} & 8,640 \\
BatchNorm2d-104 & {$[-1,960,8,8]$} & 1,920 \\
Conv2d-105 & {$[-1,160,8,8]$} & 153,600 \\
BatchNorm2d-106 & {$[-1,160,8,8]$} & 320 \\
BaseBlock-107 & {$[-1,160,8,8]$} & 0 \\
Conv2d-108 & {$[-1,960,8,8]$} & 153,600 \\
BatchNorm2d-109 & {$[-1,960,8,8]$} & 1,920 \\
Conv2d-110 & {$[-1,960,8,8]$} & 8,640 \\
BatchNorm2d-111 & {$[-1,960,8,8]$} & 1,920 \\
Conv2d-112 & {$[-1,160,8,8]$} & 153,600 \\
BatchNorm2d-113 & {$[-1,160,8,8]$} & 320 \\
BaseBlock-114 & {$[-1,160,8,8]$} & 0 \\
Conv2d-115 & {$[-1,960,8,8]$} & 153,600 \\
BatchNorm2d-116 & {$[-1,960,8,8]$} & 1,920 \\
Conv2d-117 & {$[-1,960,8,8]$} & 8,640 \\
BatchNorm2d-118 & {$[-1,960,8,8]$} & 1,920 \\
Conv2d-119 & {$[-1,320,8,8]$} & 307,200 \\
BatchNorm2d-120 & {$[-1,320,8,8]$} & 640 \\
BaseBlock-121 & {$[-1,320,8,8]$} & 0 \\
Conv2d-122 & {$[-1,1280,8,8]$} & 409,600 \\
BatchNorm2d-123 & {$[-1,1280,8,8]$} & 2,560 \\
Linear-124 & {$[-1,10]$} & 12,810 \\
\hline
\end{tabular}

TABLE V: Summary of Baseline

\begin{tabular}{|l|l|l|}
\hline Layer & output Shape & Param \# \\
\hline Conv2d-1 & {$[-1,32,32,32]$} & 864 \\
BatchNorm2d-2 & {$[-1,32,32,32]$} & 64 \\
Conv2d-3 & {$[-1,32,32,32]$} & 1,024 \\
BatchNorm2d-4 & {$[-1,32,32,32]$} & 64 \\
Conv2d-5 & {$[-1,32,32,32]$} & 288 \\
BatchNorm2d-6 & {$[-1,32,32,32]$} & 64 \\
Conv2d-7 & {$[-1,16,32,32]$} & 512 \\
BatchNorm2d-8 & {$[-1,16,32,32]$} & 32 \\
BaseBlock-9 & {$[-1,16,32,32]$} & 0 \\
Conv2d-10 & {$[-1,24,32,32]$} & 864 \\
Conv2d-11 & {$[-1,24,32,32]$} & 384 \\
HetConv-12 & {$[-1,24,32,32]$} & 0 \\
Conv2d-13 & {$[-1,24,32,32]$} & 1,296 \\
Conv2d-14 & {$[-1,24,32,32]$} & 576 \\
HetConv-15 & {$[-1,24,32,32]$} & 0 \\
Conv2d-16 & {$[-1,32,32,32]$} & 1,728 \\
Conv2d-17 & {$[-1,32,32,32]$} & 768 \\
HetConv-18 & {$[-1,32,32,32]$} & 0 \\
Conv2d-19 & {$[-1,32,32,32]$} & 2,304 \\
Conv2d-20 & {$[-1,32,32,32]$} & 1,024 \\
HetConv-21 & {$[-1,32,32,32]$} & 0 \\
Conv2d-22 & {$[-1,32,32,32]$} & 2,304 \\
\hline \multicolumn{2}{|l|}{} \\
\hline \multicolumn{2}{|l}{}
\end{tabular}

\begin{tabular}{|l|l|l|}
\hline Layer & output shape & Param $\#$ \\
\hline Conv2d-23 & {$[-1,32,32,32]$} & 1,024 \\
HetConv-24 & {$[-1,32,32,3]$} & 0 \\
Conv2d-25 & {$[-1,192,32,32]$} & 6,144 \\
BatchNorm2d-26 & {$[-1,192,32,3]$} & 384 \\
Conv2d-27 & {$[-1,192,16,16]$} & 1,728 \\
BatchNorm2d-28 & {$[-1,192,16,16]$} & 384 \\
Conv2d-29 & {$[-1,64,16,16]$} & 12,288 \\
BatchNorm2d-30 & {$[-1,64,16,16]$} & 128 \\
BaseBlock-31 & {$[-1,64,16,16]$} & 0 \\
Conv2d-32 & {$[-1,64,16,16]$} & 9,216 \\
Conv2d-33 & {$[-1,64,16,16]$} & 4,096 \\
HetConv-34 & {$[-1,64,16,16]$} & 0 \\
Conv2d-35 & {$[-1,64,16,16]$} & 9,216 \\
Conv2d-36 & {$[-1,64,16,16]$} & 4,096 \\
HetConv-37 & {$[-1,64,16,16]$} & 0 \\
Conv2d-38 & {$[-1,64,16,16]$} & 9,216 \\
Conv2d-39 & {$[-1,64,16,16]$} & 4,096 \\
HetConv-40 & {$[-1,64,16,16]$} & 0 \\
Conv2d-41 & {$[-1,96,16,16]$} & 13,824 \\
Conv2d-42 & {$[-1,96,16,16]$} & 6,144 \\
HetConv-43 & {$[-1,96,16,16]$} & 0 \\
Conv2d-44 & {$[-1,96,16,16]$} & 20,736 \\
Conv2d-45 & {$[-1,96,16,16]$} & 9,216 \\
HetConv-46 & {$[-1,96,16,16]$} & 0 \\
Conv2d-47 & {$[-1,96,16,16]$} & 20,736 \\
Conv2d-48 & {$[-1,96,16,16]$} & 9,216 \\
HetConv-49 & {$[-1,96,16,16]$} & 0 \\
Conv2d-50 & {$[-1,576,16,16]$} & 55,296 \\
BatchNorm2d-51 & {$[-1,576,16,16]$} & 1,152 \\
Conv2d-52 & {$[-1,576,8,8]$} & 5,184 \\
BatchNorm2d-53 & {$[-1,576,8,8]$} & 1,152 \\
Conv2d-54 & {$[-1,160,8,8]$} & 92,160 \\
BatchNorm2d-55 & {$[-1,160,8,8]$} & 320 \\
BaseBlock-56 & {$[-1,160,8,8]$} & 0 \\
Conv2d-57 & {$[-1,160,8,8]$} & 57,600 \\
Conv2d-58 & {$[-1,160,8,8]$} & 25,600 \\
HetConv-59 & {$[-1,160,8,8]$} & 0 \\
Conv2d-60 & {$[-1,160,8,8]$} & 57,600 \\
Conv2d-61 & {$[-1,160,8,8]$} & 25,600 \\
HetConv-62 & {$[-1,160,8,8]$} & 0 \\
Conv2d-63 & {$[-1,320,8,8]$} & 115,200 \\
Conv2d-64 & {$[-1,320,8,8]$} & 51,200 \\
HetConv-65 & {$[-1,320,8,8]$} & 0 \\
Conv2d-66 & {$[-1,1280,8,8]$} & 409,600 \\
BatchNorm2d-67 & 2,560 \\
Linear-68 & {$[-10]$} & 12,810 \\
\hline
\end{tabular}

TABLE VI: Summary of RMNv2 